Research Article

\title{
Target-Induced Aggregation of Gold Nanoparticles for Colorimetric Detection of Bisphenol A
}

\author{
Sung Hyun Hwang, ${ }^{1}$ Sehan Jeong, ${ }^{1}$ Hyung Joo Choi, ${ }^{1}$ Hyunmin Eun, ${ }^{1}$ Min Geun Jo, ${ }^{1}$ \\ Woo Young Kwon, ${ }^{1}$ Seokjoon Kim, ${ }^{1}$ Yonghwan Kim, ${ }^{2}$ Miran Lee, ${ }^{2}$ and Ki Soo Park $\mathbb{D}^{1}$ \\ ${ }^{1}$ Department of Biological Engineering, College of Engineering, Konkuk University, Seoul 05029, Republic of Korea \\ ${ }^{2}$ Daisung Green Tech, Seongnam, Gyeonggi-do 13216, Republic of Korea \\ Correspondence should be addressed to Ki Soo Park; kskonkuk@gmail.com
}

Received 20 February 2019; Revised 10 July 2019; Accepted 1 August 2019; Published 8 September 2019

Academic Editor: Ilaria Fratoddi

Copyright (c) 2019 Sung Hyun Hwang et al. This is an open access article distributed under the Creative Commons Attribution License, which permits unrestricted use, distribution, and reproduction in any medium, provided the original work is properly cited.

\begin{abstract}
Bisphenol A (BPA) is used in a wide variety of consumer products owing to its beneficial properties of optical clarity, shatter resistance, and heat resistance. However, leached BPA has been shown to disturb the endocrine system and could cause cancer even at low concentrations, which has led to public concern. To reduce the toxic effects caused by BPA, it is important to monitor the BPA levels and its presence in products in a simple, rapid, and on-site manner. Here, we propose a new colorimetric strategy for the simple and rapid detection of BPA employing a DNA aptamer, a cationic surfactant, and gold nanoparticles (AuNPs). Using the developed system, the presence of BPA can be successfully determined based simply on a visually detectable color change from red to blue, triggered by aggregate formation of the AuNPs, which can be monitored even with the naked eye. Under the optimized conditions, this system could detect BPA with excellent selectivity and sensitivity, and its high performance was validated in the receipt obtained from local market and BPA-spiked tap water samples, ensuring its practical applicability. Moreover, the limit of the detection of the system was determined to be $97 \mathrm{nM}$, which is below the current tolerable daily intake level, demonstrating its suitability for toxicity assessment and on-site quality control in a more economical manner when compared with conventional methods.
\end{abstract}

\section{Introduction}

Bisphenol A (BPA) is a widely used material for diverse consumer products as the main constituent of polycarbonate plastics and epoxy resins, including CDs, food storage containers, paints, and protective coatings, owing to its beneficial properties of optical clarity, shatter resistance, and heat resistance [1]. BPA was first approved by the Food and Drug Administration in the 1960s, but concerns have been raised in recent decades about its possible negative impact on human health [2]. In particular, BPA leached from the product has estrogen-mimicking properties and can thus bind to estrogen receptors, thereby interfering with the endocrine system $[3,4]$. In addition, BPA has been associated with carcinogenesis and can interfere with the immune response and nervous system through various cell signaling pathways [5-7]. Thus, the European Food Safety Authority estab- lished the tolerable daily intake (TDI) at $0.05 \mathrm{mg} \mathrm{BPA} / \mathrm{kg}$ body weight per day [8].

Accordingly, it is essential to develop effective methods for preventing the toxic effects of BPA. The conventional methods rely on chromatography-based techniques, such as high-performance liquid chromatography (HPLC) $[9,10]$ and liquid chromatography mass spectrometry [11, 12], and enzyme-based method such as enzyme-linked immunosorbent assay [13]. However, these methods are limited for on-site application owing to the bulky instrumentation and expensive enzymes required; thus, extensive effort has been paid to developing simpler and more economical alternative BPA detection methods. Notably, colorimetric strategies that enable rapid and immediate visual detection of the presence of BPA have received special attention [14,15]. Representative examples of such methods utilize DNA aptamers, which are in vitro selected single-stranded DNAs that specifically 
bind to BPA and gold nanoparticles (AuNPs) $[16,17]$. In addition, different types of chemicals such as conjugated polymers and high concentrations of salts are used to regulate the assembly of AuNPs [18-21], which leads to the transition from a dispersed (red color) state to an aggregated (blue color) state. However, they still have drawbacks such as the requirement of the expensive conjugated polymers or vulnerability to the false-positive/negative signals.

In this study, we expanded the aptamer-based colorimetric strategy for the effective analysis of BPA using the cationic surfactant, cetrimonium bromide (CTAB). As compared to previous approaches in which the chemicals such as conjugated polymers and salts only serve as the aggregation agents for AuNPs, the positively charged head and hydrophobic tails in a single CTAB molecule allow for the direct induction of the aggregation of AuNPs while also interacting with DNA aptamers [22]. More importantly, CTAB is a relatively inexpensive and widely available reagent, which forms supramolecules with DNA aptamers only in the absence of target molecules [23], thereby effectively preventing the aggregation of AuNPs to result in a low background signal and facilitate sensitive and specific detection [24]. Using this proposed system, we successfully determined the presence of BPA in less than $20 \mathrm{~min}$ based on the direct color change that could be detected with the naked eye. In addition, we validated the practical applicability of the method by detecting BPA spiked in receipt and tap water.

\section{Materials and Methods}

2.1. Preparation of AuNPs. AuNPs were synthesized by reducing chloroauric acid $\left(\mathrm{HAuCl}_{4}\right)$ with sodium citrate (Sigma, Korea). In brief, all glassware was first immersed in aqua regia solution, a mixture of hydrochloric acid $(\mathrm{HCl})$ and nitric acid $\left(\mathrm{HNO}_{3}\right)$ (Sigma, Korea) at a molar ratio of $3: 1$, and rinsed with distilled water. Next, the $\mathrm{HAuCl}_{4}$ solution $(1 \mathrm{mM})$ was mixed with sodium citrate $(38.8 \mathrm{mM})$ under vigorous stirring and boiling at $100^{\circ} \mathrm{C}$ for $13 \mathrm{~min}$ until the color changed to wine-red. The resulting AuNPs whose concentration was $4.42 \times 10^{12}$ particles $/ \mathrm{mL}$ (denoted as " $1 \mathrm{X}$ ") were stored at $4^{\circ} \mathrm{C}$ before use. The size of AuNPs was determined to be $20 \mathrm{~nm}$ using dynamic light scattering (DLS) (DynaPro Plate Reader, Wyatt Technology, USA) (Figure S1).

2.2. Optimization of CTAB, DNA Aptamer, and AuNP Concentrations. Different concentrations of CTAB and other surfactants including dodecyltrimethylammonium bromide (DTAB), cetylpyridinium chloride (CPC), and sodium dodecyl sulfate (SDS) (Sigma, Korea) were tested to find the optimal concentration at which the AuNPs are effectively aggregated. After mixing the presynthesized AuNPs (0.2X) with different concentrations of CTAB in a reaction buffer (70 mM HEPES, pH 7.4; Sigma, Korea), the absorbance values were measured at $520 \mathrm{~nm}$ and $650 \mathrm{~nm}$ on a SpectraMax iD5 multimode microplate reader (Molecular Devices, USA). Once the optimal concentration of CTAB $(4 \mu \mathrm{M})$ was determined, various concentrations of DNA aptamers were tested with this fixed concentration. The BPA-specific
DNA aptamer $\left(5^{\prime}\right.$-GGA TAG CGG GTT CC-3') [25] and the control DNA $\left(5^{\prime}\right.$-TTT TTT TTT TTA TTT TTT TTT TTT AAG CTG GGA GAA AGA AAT GGA A-3') (synthesized by Bioneer, Korea) were incubated with CTAB $(4 \mu \mathrm{M})$ for $10 \mathrm{~min}$, and AuNPs $(0.2 \mathrm{X})$ were applied. The resulting absorbance values were recorded at $520 \mathrm{~nm}$ and $650 \mathrm{~nm}$ on a microplate reader. In addition, the optimal concentrations of AuNPs, CTAB, and DNA aptamer were also found by evaluating the responses of the developed system in the absence and presence of BPA $(50 \mu \mathrm{M})$ (Figure S2).

2.3. Detection Feasibility, Sensitivity, and Selectivity. To determine the specificity of the system for BPA detection, $100 \mathrm{nM}$ of the DNA aptamer was mixed with different concentrations of BPA or other chemicals with a similar structure to BPA, including diclofenac, tyrosine, tryptophan, and phenylalanine (all from Samchun Chemical, Korea), in reaction buffer (70 mM HEPES, pH 7.4), and incubated for $10 \mathrm{~min}$ at room temperature, followed by the addition of $4 \mu \mathrm{M}$ of CTAB and incubation for $10 \mathrm{~min}$. The presynthesized AuNPs were then added to the mixtures, and the resulting absorbance values were immediately recorded at $520 \mathrm{~nm}$ and $650 \mathrm{~nm}$ on the microplate reader. All absorbance data are presented as the $A_{650} / A_{520}$ ratio.

2.4. Tap Water Spiking Test. Mock samples containing BPA were prepared by spiking different concentrations of BPA in tap water $(10 \%)$ and analyzed in the same manner described above. For recovery determination, the standard curve in tap water (10\%) was constructed and used to detect unknown concentrations of BPA in a sample.

2.5. Detection of BPA in the Receipt. The receipt $(0.86 \mathrm{~g}$, $8 \times 16 \mathrm{~cm}$ ) obtained from the local market was first cut into some pieces. Next, the receipt was dissolved in $100 \mathrm{~mL}$ of distilled water, which was heated at $100^{\circ} \mathrm{C}$ for $10 \mathrm{~min}$. Finally, the solution was filtered by a $0.2 \mu \mathrm{m}$ filter (Sartorius Stedim, Korea) to remove the large particles, which was then subjected to the detection procedure.

\section{Results and Discussion}

3.1. Colorimetric Detection of BPA. The scheme for the colorimetric detection of BPA is illustrated in Figure 1. The color change is based on the target (BPA)-induced aggregation of AuNPs. In the absence of BPA, a DNA aptamer that specifically binds to BPA is retained in its native structure, exhibiting a negative surface charge. Thus, CTAB, as a cationic surfactant, interacts with the DNA aptamer by forming supramolecules, leaving AuNPs in a dispersed state [26, 27]. In contrast, in the presence of BPA, BPA takes up the aptamers to form aptamer-BPA complexes, so that CTAB interacts with the negatively charged AuNPs, leading to their aggregation [28]. Consequently, assay samples that do not contain BPA display a red color, whereas those containing BPA change to a blue color, which can be easily distinguished by the naked eye.

3.2. Optimization of CTAB, DNA Aptamer, and AuNP Concentrations. Before determining the feasibility of this 


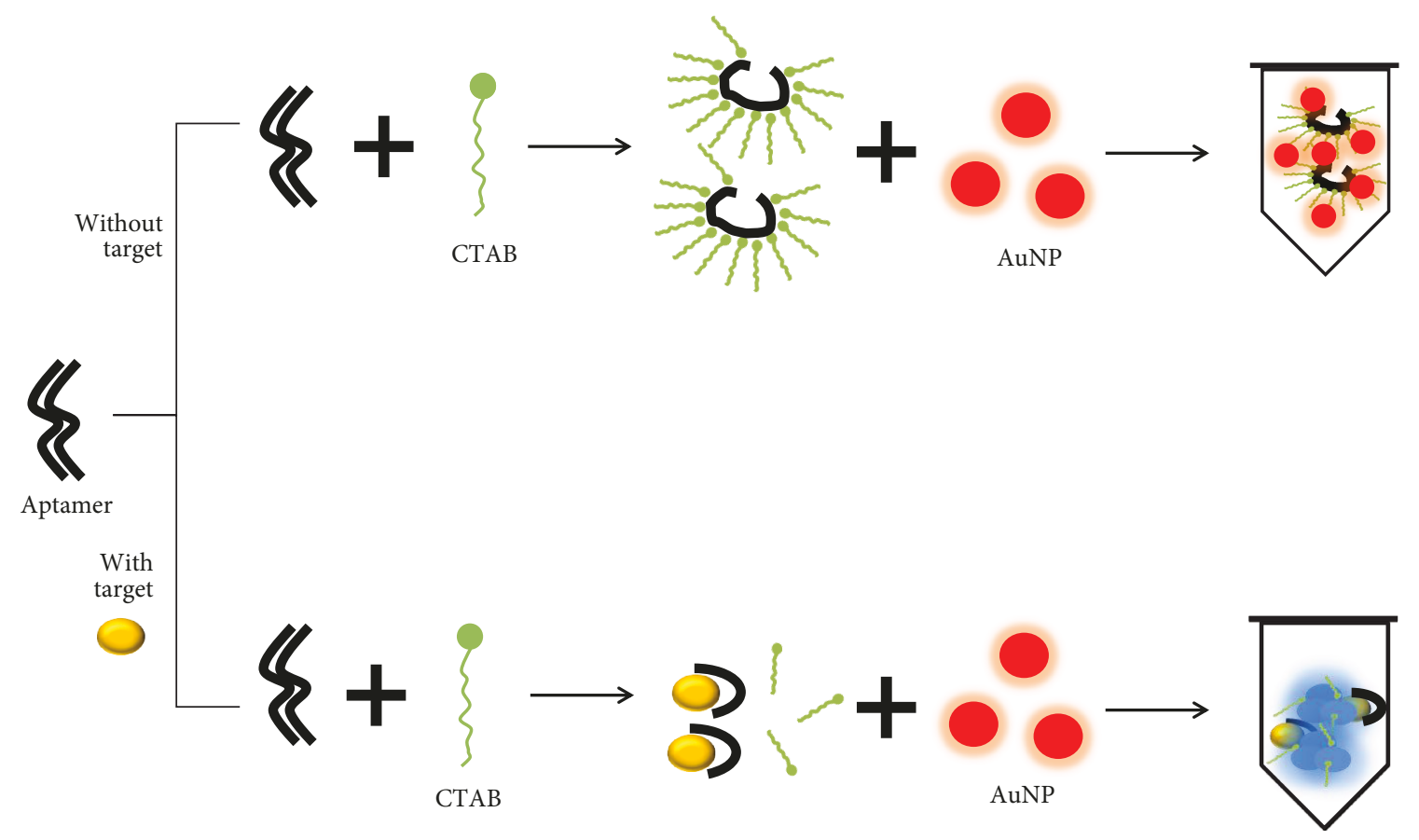

FIGURE 1: Schematic illustration (not drawn to scale) of colorimetric detection of BPA based on the target-induced aggregation of AuNPs.

concept for BPA detection, we first optimized the concentrations of CTAB and DNA aptamers, the key factors required for successful operation of the developed system. We assumed that the discrepancy between the concentrations of CTAB and DNA aptamers could cause false-positive and negative signals. For the quantitative analysis of the colorimetric response, the $A_{650}$ to $A_{520}$ ratio, corresponding to wavelengths of the aggregated (blue color) and dispersed (red color) AuNP states, respectively, was spectrophotometrically determined, in which a higher ratio indicates transition from a dispersed state to an aggregated state, i.e., the presence of BPA [29].

As shown in Figure 2(a), with increasing CTAB concentration, the $A_{650} / A_{520}$ ratio increased; since AuNPs were completely aggregated with $4 \mu \mathrm{M}$ CTAB, this concentration was selected for further experiments. We then optimized the concentration of the DNA aptamer using $4 \mu \mathrm{M}$ CTAB. In line with the concept demonstrated in the schematic illustration (Figure 1), the DNA aptamer interacted with CTAB to form supramolecules, thereby preventing the aggregation of AuNPs (Figure 2(b)). In addition, the responses of the proposed method in the absence and presence of BPA were evaluated by changing the concentration of AuNPs, CTAB, and DNA aptamer. The results in Figure S2 show that $0.2 \mathrm{X}$ of AuNPs, $4 \mu \mathrm{M}$ of CTAB, and $100 \mathrm{nM}$ of DNA aptamer are ideal to achieve the highest signal changes in the presence of BPA, which were used for further experiments. It was also confirmed that $\mathrm{CTAB}$ is the most effective to generate the colorimetric response in the presence of BPA (Figure S3).

3.3. Feasibility of BPA Detection with the Developed System. The detection feasibility of the developed system was then investigated under the optimized conditions. As shown in the absorbance spectra in Figure 3(a), the sample solution without BPA exhibited a maximum absorbance peak at $520 \mathrm{~nm}$, the characteristic wavelength of dispersed AuNPs (red color). By contrast, the sample solution containing BPA showed the maximum absorbance peak at $650 \mathrm{~nm}$, the characteristic wavelength of aggregated AuNPs (blue color). In addition, control DNA that does not bind to BPA was tested to confirm the specificity of the interaction of the DNA aptamer with BPA. As expected, the sample solutions did not show a change from red to blue using the control aptamer, as further evidenced by the maximum absorbance at $520 \mathrm{~nm}$ with no significant change in absorbance regardless of the presence of BPA (Figure S4). Overall, these results confirmed our hypothesis that the target (BPA) binds to the DNA aptamer and, thus, CTAB cannot interact with the DNA aptamer, which induces the aggregation of AuNPs accompanied by a color change from red to blue, ensuring the detection feasibility of this strategy.

3.4. Detection Sensitivity and Selectivity of the Developed System. Next, we determined the sensitivity of the system by measuring the $A_{650} / A_{520}$ ratio of samples containing different known concentrations of BPA. As shown in Figure $4(\mathrm{a})$, the $A_{650} / A_{520}$ ratio increased linearly with increasing concentrations of BPA $\left(R^{2}=0.9807\right)$ in the range of 0-50 $\mu \mathrm{M}$, demonstrating the linear equation $A_{650} / A_{520}=$ $0.013 \times C_{\mathrm{BPA}}+0.4703$, where $C_{\mathrm{BPA}}$ is the BPA concentration. Based on the definition of the limit of detection (LOD), $3 \sigma / S$, where $\sigma$ and $S$ are the standard deviation of the blank sample and the slope of the linear relationship, respectively, the LOD was calculated to be $97 \mathrm{nM}$. This value is comparable or slightly inferior to those obtained for BPA detection with conventional methods (Table S1) but is sufficiently low to detect BPA levels below the current TDI $(1500 \mu \mathrm{g} / \mathrm{L}=6.57 \mu \mathrm{M}$, calculated on the basis 


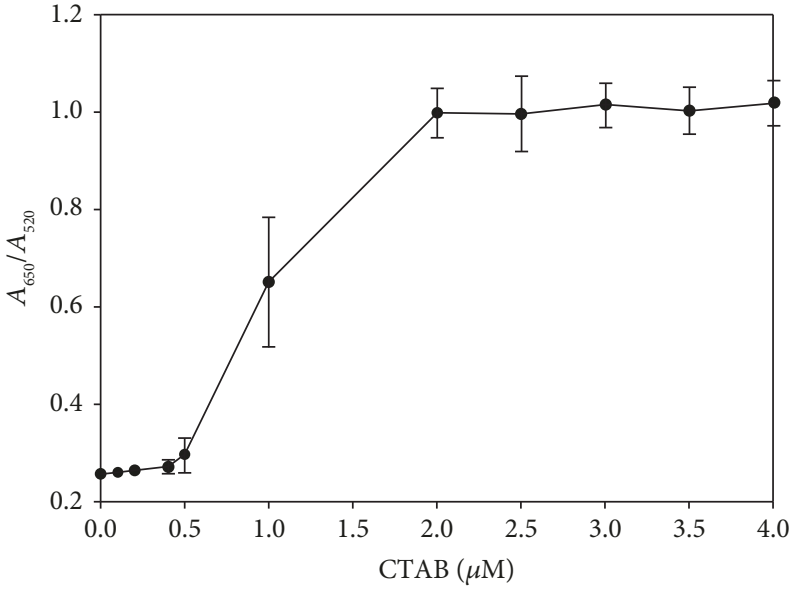

(a)

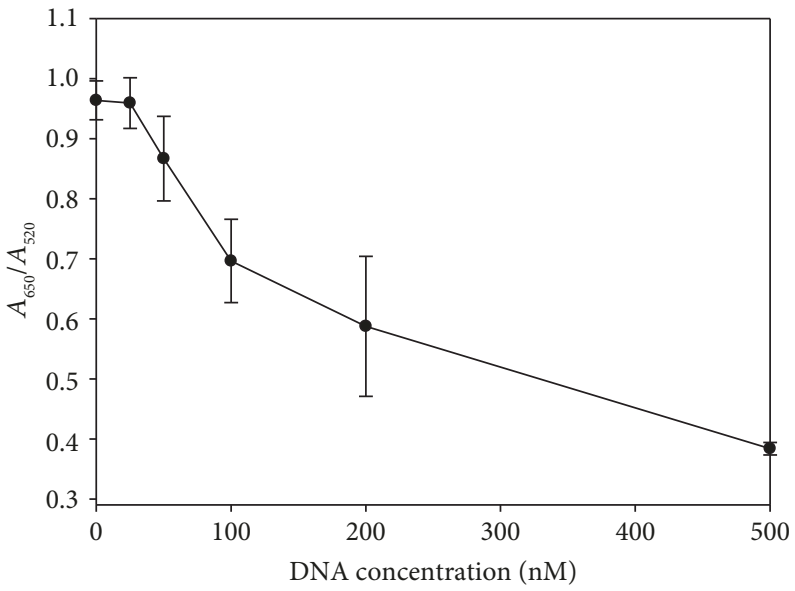

(b)

FIgURE 2: Optimization of the concentrations of CTAB (a) and DNA aptamer (b) for successful operation of the developed system. (a) The final concentration of AuNPs was $0.2 \mathrm{X}$. (b) The final concentrations of AuNPs and CTAB were $0.2 \mathrm{X}$ and $4 \mu \mathrm{M}$, respectively.

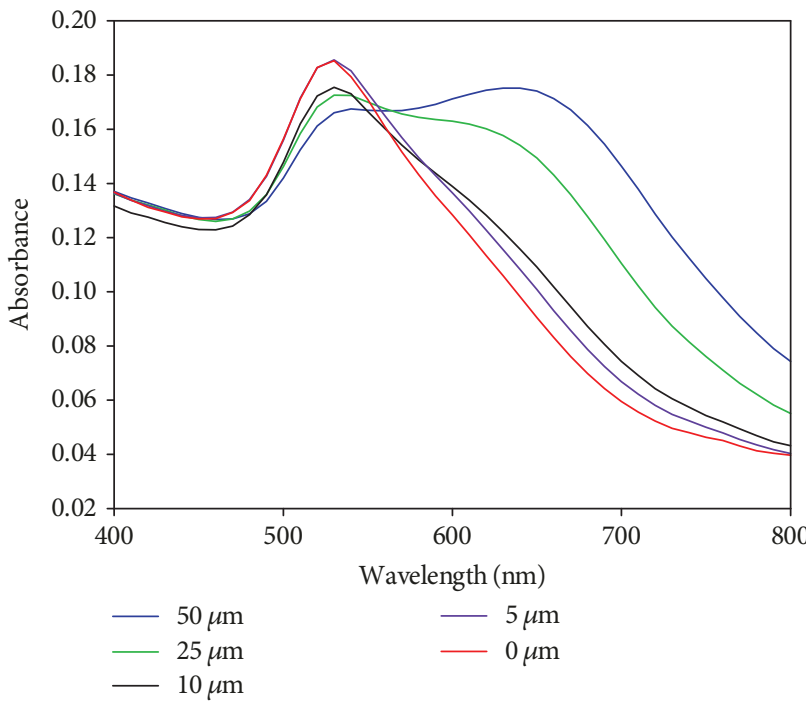

(a)

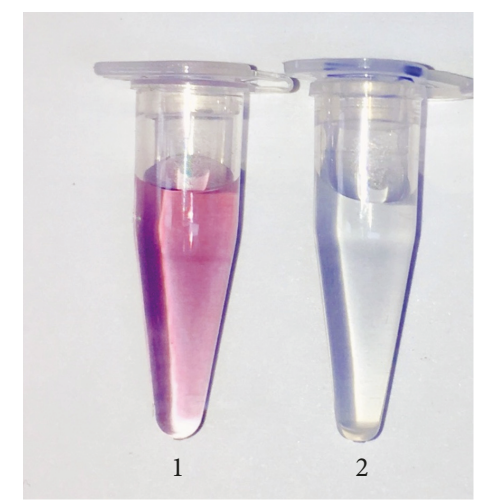

(b)

Figure 3: Detection feasibility of the developed system. (a) Absorbance spectra of the sample solutions without $(0 \mu \mathrm{M})$ and with BPA at different concentrations $(5,10,25$, and $50 \mu \mathrm{M})$. (b) Photographs of the sample solutions (1: without BPA $(0 \mu \mathrm{M})$; 2: with BPA $(25 \mu \mathrm{M})$ ). The final optimized concentrations of AuNPs, DNA aptamer, and CTAB were $0.2 \mathrm{X}, 100 \mathrm{nM}$, and $4 \mu \mathrm{M}$, respectively.

of the normally assumed $60 \mathrm{~kg}$ body weight) $[14,30]$. However, the particular advantage of the newly developed system is that it produces the visual colorimetric response in less than $20 \mathrm{~min}$ and does not require expensive instrumentation as in HPLC-based detection.

The selectivity of the method was also evaluated by employing chemicals that have aromatic groups like those in BPA (tyrosine, tryptophan, and phenylalanine) in addition to another harmful chemical (diclofenac). Figure 4(b) shows that only solutions containing BPA caused the aggregation of AuNPs, as manifested by the high $A_{650} / A_{520}$ ratio, while no noticeable increases in the ratio were observed in the presence of the other chemicals. These results proved that this system is highly selective to BPA, supporting that the specific interaction of the DNA aptamer with BPA induces the CTAB-mediated aggregation of AuNPs.

3.5. Detection of BPA Spiked in Tap Water. Finally, we verified the practical applicability of the proposed system by determining the BPA content in spiked tap water. Specifically, mock samples were prepared by spiking different concentrations of BPA into tap water (10\%). As shown in Figure S5, an excellent linear relationship $\left(R^{2}=0.9799\right)$ was observed between $A_{650} / A_{520}$ and $C_{\mathrm{BPA}}$ in the tap water samples. Based on this calibration curve, we determined the concentrations of BPA in tap water with high reproducibility and precision, as evidenced by the coefficients of variations and recovery rates for samples containing both low $(5 \mu \mathrm{M})$ and high 


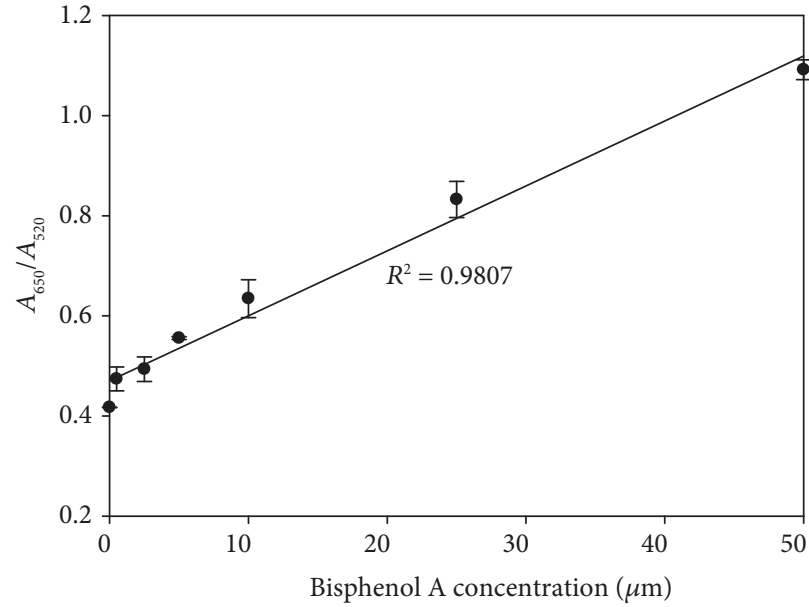

(a)

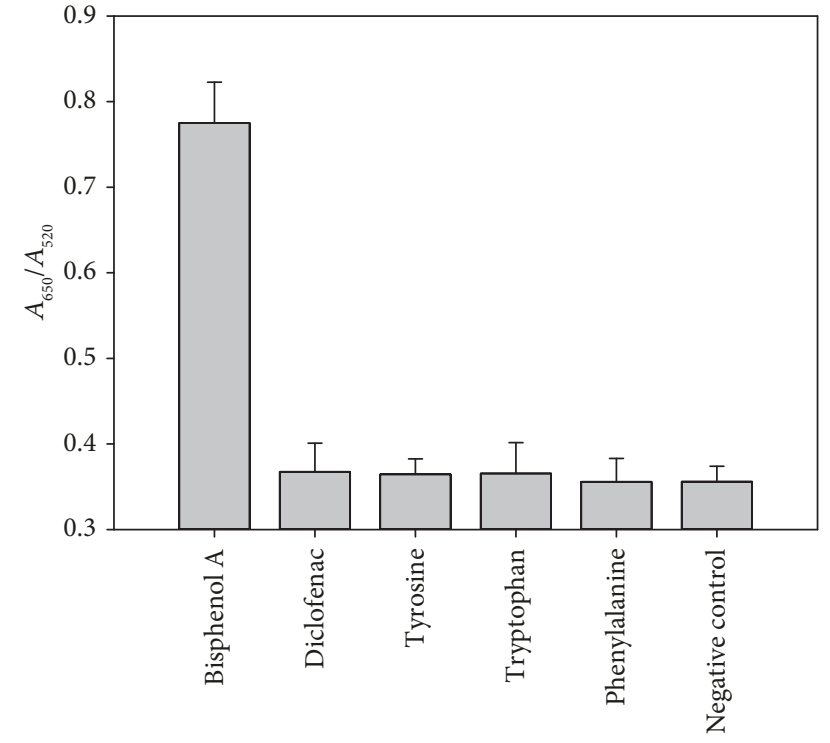

(b)

FIGURE 4: Detection sensitivity (a) and selectivity (b) of the developed system. The final optimized concentrations of AuNPs, DNA aptamer, and CTAB used were $0.2 \mathrm{X}, 100 \mathrm{nM}$, and $4 \mu \mathrm{M}$, respectively. BPA and other chemicals (diclofenac, tyrosine, tryptophan, and phenylalanine) were used at $25 \mu \mathrm{M}$ each.

TABLE 1: Determination of BPA in the receipt.

\begin{tabular}{lccccc}
\hline Determined BPA $(\mu \mathrm{M})^{\mathrm{a}}$ & Amount of BPA added $(\mu \mathrm{M})$ & Measured BPA $(\mu \mathrm{M})$ & SD $^{\mathrm{c}}$ & $\mathrm{CV}^{\mathrm{d}}(\%)$ & Recovery $^{\mathrm{e}}(\%)$ \\
\hline 2.51 & 5.00 & 5.49 & 0.735 & 13.4 & 110 \\
& 25.0 & 27.9 & 0.067 & 0.241 & 112 \\
\hline
\end{tabular}

This concentration is determined by diluting the receipt solution $\left(100 \mathrm{~mL}\right.$; See Materials and Methods) 20 times. ${ }^{\mathrm{b}}$ Mean of three measurements. ${ }^{\mathrm{C}} \mathrm{Standard}$ deviation (SD) of three measurements. ${ }^{\mathrm{d}}$ Coefficient of variation $(\mathrm{CV})=(\mathrm{SD} / \mathrm{mean}) \times 100(\%) .{ }^{\mathrm{e}}$ Recovery $=($ mean $/$ amount added $) \times 100(\%)$.

$(15 \mu \mathrm{M})$ concentrations of BPA (Table S2). These results confirm that the developed system enables the reliable detection of BPA in real samples [18, 19].

3.6. Detection of BPA in the Receipt. Even though the results in the tap water were promising, the tap water is relatively pure and does not contain many impurities, which is hard to reflect the real practical situation. Thus, we attempted to check the amount of BPA in the receipt obtained from the local market. As shown in Figure S6, the $A_{650} / A_{520}$ ratio increased linearly with increasing concentration of BPA additionally spiked in the receipt solution (See Materials and Methods for details) and the concentration of BPA present in the receipt was determined to be $2.51 \mu \mathrm{M}$ by the standard addition method (Table 1) [31]. In addition, varying concentrations of added BPA in the receipt solution were accurately measured, as evidenced by the coefficients of variations $(13.4 \%$ and $0.241 \%$ ) and recovery rates $(110 \%$ and $112 \%)$.

\section{Conclusions}

We developed a colorimetric strategy for the rapid and simple detection of BPA based on the target-induced aggregation of AuNPs. For the effective transition from a dispersed state to an aggregated state of AuNPs in the presence of the target
(BPA), the concentrations of the BPA-specific DNA aptamer and a cationic surfactant, CTAB, were systematically optimized. The presence of BPA results in complex formation with the DNA aptamer, which frees CTAB to induce the aggregation of AuNPs, resulting in the transition from red to blue-colored solution. Importantly, this is the first report to propose a colorimetric system for the detection of a small molecule, BPA, based on the CTAB-mediated aggregation of AuNPs. With the developed system that produces a colorimetric signal that can be detected by the naked eye, we successfully detected BPA in less than $20 \mathrm{~min}$, and its sensitivity, selectivity, and practical applicability were demonstrated. We expect that the developed system will be applied to point-of-care settings, enabling the on-site detection of BPA and consequently reducing the concerns related to its toxicity.

\section{Data Availability}

The data can be made available from the corresponding author upon reasonable request.

\section{Conflicts of Interest}

The authors declare that there are no conflicts of interest regarding the publication of this paper. 


\section{Authors' Contributions}

Sung Hyun Hwang and Sehan Jeong equally contributed to this work.

\section{Acknowledgments}

This work was supported by the Korea Environment Industry \& Technology Institute (KEITI) through Public Technology Program based on Environmental Policy, funded by the Korean Ministry of Environment (MOE) (2016000200008).

\section{Supplementary Materials}

Table S1: comparison of this method with other methods for the detection of BPA. Table S2: determination of BPA in tap water. Figure S1: size distribution of the prepared AuNPs. Figure S2: optimization of the concentrations of AuNPs, CTAB, and BPA-specific DNA aptamer. Figure S3: optimization of the types of surfactants. Figure S4: absorbance spectra of the control sample solutions. Figure S5: linear relationship between $A_{650} / A_{520}$ and the concentration of BPA in tap water (10\%). Figure S6: standard addition method used for the determination of BPA in a receipt solution. (Supplementary Materials)

\section{References}

[1] E. Ribeiro, C. Ladeira, and S. Viegas, "Occupational exposure to bisphenol A (BPA): a reality that still needs to be unveiled," Toxics, vol. 5, no. 3, p. 22, 2017.

[2] K. L. Hernandez-Hernandez, N. Tapia-Orozco, M. Gimeno et al., "Exposure to bisphenol A: current levels from food intake are toxic to human cells," Molecular Biology Reports, vol. 46, no. 2, pp. 2555-2559, 2019.

[3] L. N. Vandenberg, M. V. Maffini, C. Sonnenschein, B. S. Rubin, and A. M. Soto, "Bisphenol-A and the great divide: a review of controversies in the field of endocrine disruption," Endocrine Reviews, vol. 30, no. 1, pp. 75-95, 2009.

[4] B. S. Rubin, "Bisphenol-A: an endocrine disruptor with widespread exposure and multiple effects," Journal of Steroid Biochemistry and Molecular Biology, vol. 127, no. 1-2, pp. 27-34, 2011.

[5] S. Li, Y. Jin, H. Zhao, Y. Jiang, and Z. Cai, "Evaluation of bisphenol A exposure induced oxidative RNA damage by liquid chromatography-mass spectrometry," Chemosphere, vol. 222, pp. 235-242, 2019.

[6] M. Murata and J. H. Kang, "Bisphenol A (BPA) and cell signaling pathways," Biotechnology Advances, vol. 36, no. 1, pp. 311-327, 2018.

[7] D. D. Seachrist, K. W. Bonk, S. M. Ho, G. S. Prins, A. M. Soto, and R. A. Keri, "A review of the carcinogenic potential of bisphenol A," Reproductive Toxicology, vol. 59, pp. 167-182, 2016.

[8] S. Almeida, A. Raposo, M. Alemida-Gonzalez, and C. Carrascosa, "Bisphenol A: food exposure and impact on human health," Comprehensive Reviews in Food Science and Food Safety, vol. 17, no. 6, pp. 1503-1517, 2018.

[9] A. M. El-Kosasy, O. Abdel-Aziz, M. F. Ayad, and O. M. Mabrouk, "HPLC method for simultaneous determination of bisphenol A-diglycidyl ether and some of its reaction products in canned foods using photodiode array detector," Journal of Chromatographic Science, vol. 56, no. 10, pp. 920932, 2018.

[10] S. Li, F. Chen, F. Liu et al., "Rapid detection of bisphenol A in water samples by high-performance liquid chromatography based on syringe filters with nylon membrane extraction," Journal of Liquid Chromatography \& Related Technologies, vol. 38, no. 15, pp. 1474-1478, 2015.

[11] A. Jurek and E. Leitner, "Analytical determination of bisphenol A (BPA) and bisphenol analogues in paper products by LC-MS/MS," Food Additives \& Contaminants: Part A, vol. 35, no. 11, pp. 2256-2269, 2018.

[12] N. Dreolin, M. Aznar, S. Moret, and C. Nerin, "Development and validation of a LC-MS/MS method for the analysis of bisphenol A in polyethylene terephthalate," Food Chemistry, vol. 274, pp. 246-253, 2019.

[13] J. Zheng, S. Q. Zhao, X. T. Xu, and K. Zhang, "Detection of bisphenol $\mathrm{A}$ in water samples using ELISA determination method," Water Science and Technology: Water Supply, vol. 11, no. 1, pp. 55-60, 2011.

[14] X. Liang, H. Wang, H. Wang, and G. Pei, "Colorimetric detection of bisphenol a using $\mathrm{Au}-\mathrm{Fe}$ alloy nanoparticle aggregation," Analytical Methods, vol. 7, no. 9, pp. 39523957, 2015.

[15] Q. Kong, Y. Wang, L. Zhang, S. Ge, and J. Yu, "A novel microfluidic paper-based colorimetric sensor based on molecularly imprinted polymer membranes for highly selective and sensitive detection of bisphenol A," Sensors and Actuators B: Chemical, vol. 243, pp. 130-136, 2017.

[16] M. Jo, J. Y. Ahn, J. Lee et al., "Development of single-stranded DNA aptamers for specific bisphenol A detection," Oligonucleotides, vol. 21, no. 2, pp. 85-91, 2011.

[17] J. Xu, Y. Li, J. Bie et al., "Colorimetric method for determination of bisphenol A based on aptamer-mediated aggregation of positively charged gold nanoparticles," Microchimica Acta, vol. 182, no. 13-14, pp. 2131-2138, 2015.

[18] D. Zhang, J. Yang, J. Ye et al., "Colorimetric detection of bisphenol A based on unmodified aptamer and cationic polymer aggregated gold nanoparticles," Analytical Biochemistry, vol. 499, pp. 51-56, 2016.

[19] Y. Li, J. Xu, L. Wang et al., "Aptamer-based fluorescent detection of bisphenol A using nonconjugated gold nanoparticles and CdTe quantum dots," Sensors and Actuators B: Chemical, vol. 222, pp. 815-822, 2016.

[20] M. S. Cordray, M. Amdahl, and R. R. Richards-Kortum, "Gold nanoparticle aggregation for quantification of oligonucleotides: optimization and increased dynamic range," Analytical Biochemistry, vol. 431, no. 2, pp. 99-105, 2012.

[21] S. Christau, T. Moeller, J. Genzer, R. Koehler, and R. V. Klitzing, "Salt-induced aggregation of negatively charged gold nanoparticles confined in a polymer brush matrix," Macromolecules, vol. 50, no. 18, pp. 7333-7343, 2017.

[22] Y. Yang, S. Matsubara, M. Nogami, and J. Shi, "Controlling the aggregation behavior of gold nanoparticles," Materials Science and Engineering: B, vol. 140, no. 3, pp. 172-176, 2007.

[23] S. Lee, D. H. Manjunatha, W. Jeon, and C. Ban, "Cationic surfactant-based colorimetric detection of plasmodium lactate dehydrogenase, a biomarker for malaria, using the specific DNA aptamer," PLoS One, vol. 9, no. 7, article e100847, 2014.

[24] Y. Wu, L. Liu, S. Zhan, F. Wang, and P. Zhou, "Ultrasensitive aptamer biosensor for arsenic(III) detection in aqueous 
solution based on surfactant-induced aggregation of gold nanoparticles," Analyst, vol. 137, no. 18, pp. 4171-4178, 2012.

[25] E. H. Lee, H. J. Lim, S. D. Lee, and A. Son, "Highly sensitive detection of bisphenol A by nanoaptamer assay with truncated aptamer," ACS Applied Materials \& Interfaces, vol. 9, no. 17, pp. 14889-14898, 2017.

[26] S. Husale, W. Grange, M. Karle, S. Bürgi, and M. Hegner, "Interaction of cationic surfactants with DNA: a singlemolecule study," Nucleic Acids Research, vol. 36, no. 5, pp. 1443-1449, 2008.

[27] R. S. Dias, L.. M. Magno, A. J. M. Valente et al., "Interaction between DNA and cationic surfactants: effect of DNA conformation and surfactant headgroup," The Journal of Physical Chemistry B, vol. 112, no. 46, pp. 14446-14452, 2008.

[28] G. Du, L. Wang, D. Zhang et al., "Colorimetric aptasensor for progesterone detection based on surfactant-induced aggregation of gold nanoparticles," Analytical Biochemistry, vol. 514, pp. 2-7, 2016.

[29] C. H. Lu, Y. W. Wang, S. L. Ye, G. N. Chen, and H. H. Yang, "Ultrasensitive detection of $\mathrm{Cu} 2+$ with the naked eye and application in immunoassays," NPG Asia Materials, vol. 4, no. 3, p. e10, 2012.

[30] S. Biedermann, P. Tschudin, and K. Grob, "Transfer of bisphenol A from thermal printer paper to the skin," Analytical and Bioanalytical Chemistry, vol. 398, no. 1, pp. 571-576, 2010.

[31] K. S. Park, M. I. Kim, M. A. Woo, and H. G. Park, “A label-free method for detecting biological thiols based on blocking of $\mathrm{Hg} 2+-$ quenching of fluorescent gold nanoclusters," Biosensors and Bioelectronics, vol. 45, pp. 65-69, 2013. 


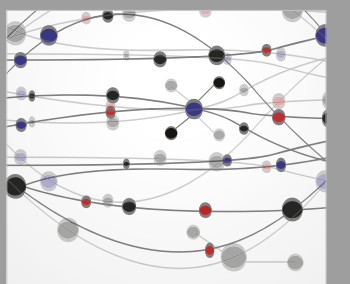

The Scientific World Journal
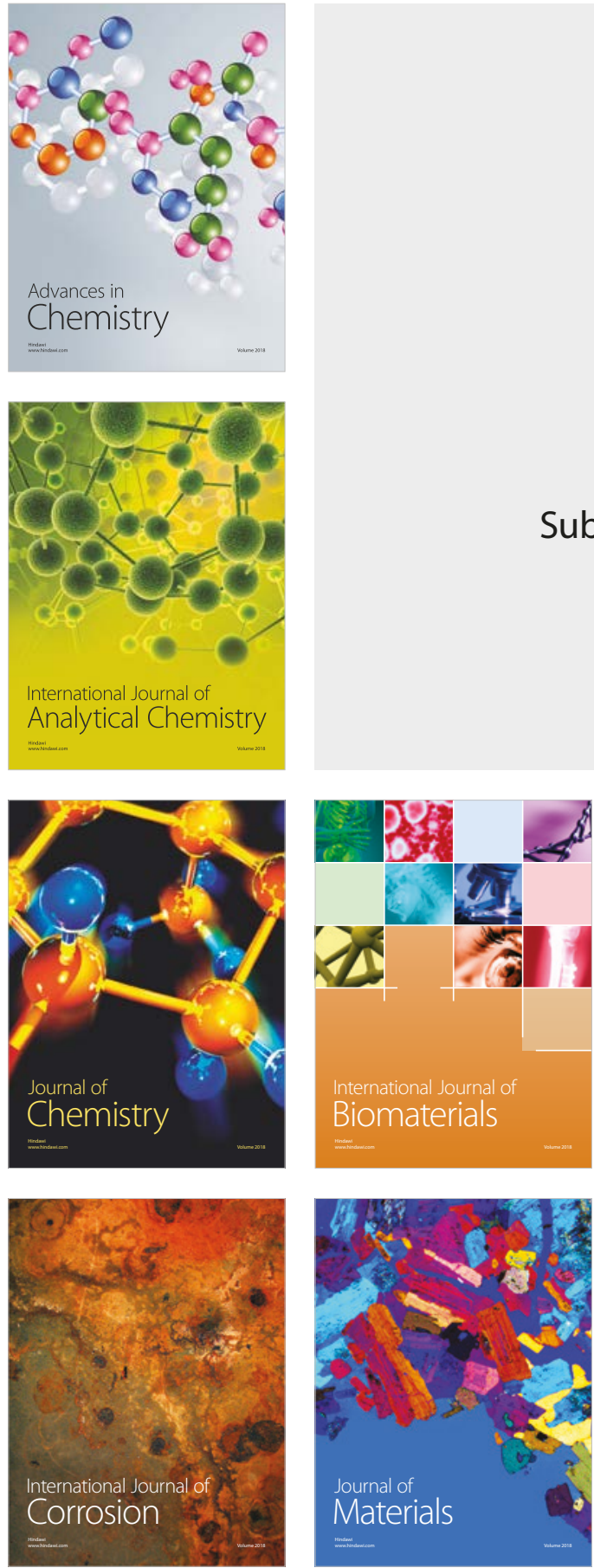

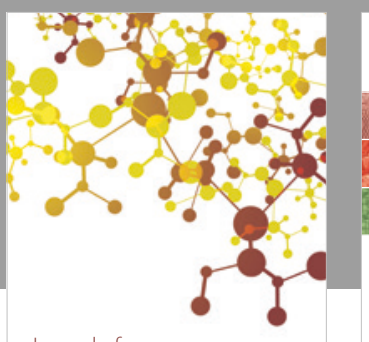

Journal of

Applied Chemistry
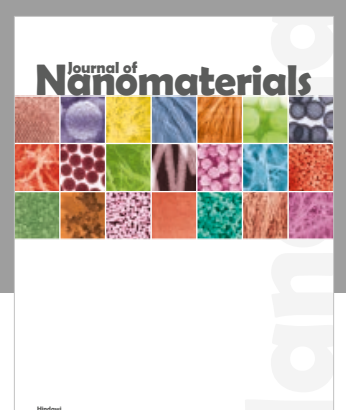

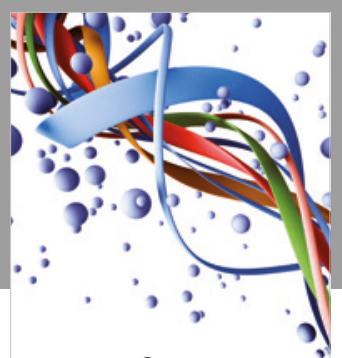

Scientifica

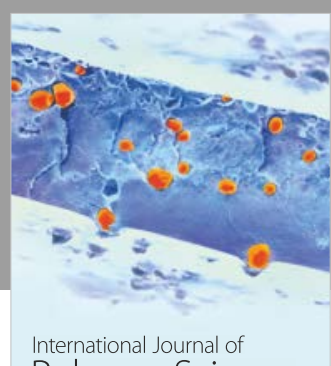

Polymer Science

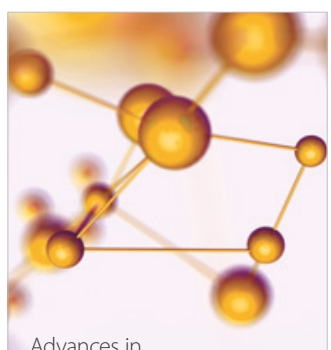

Physical Chemistry
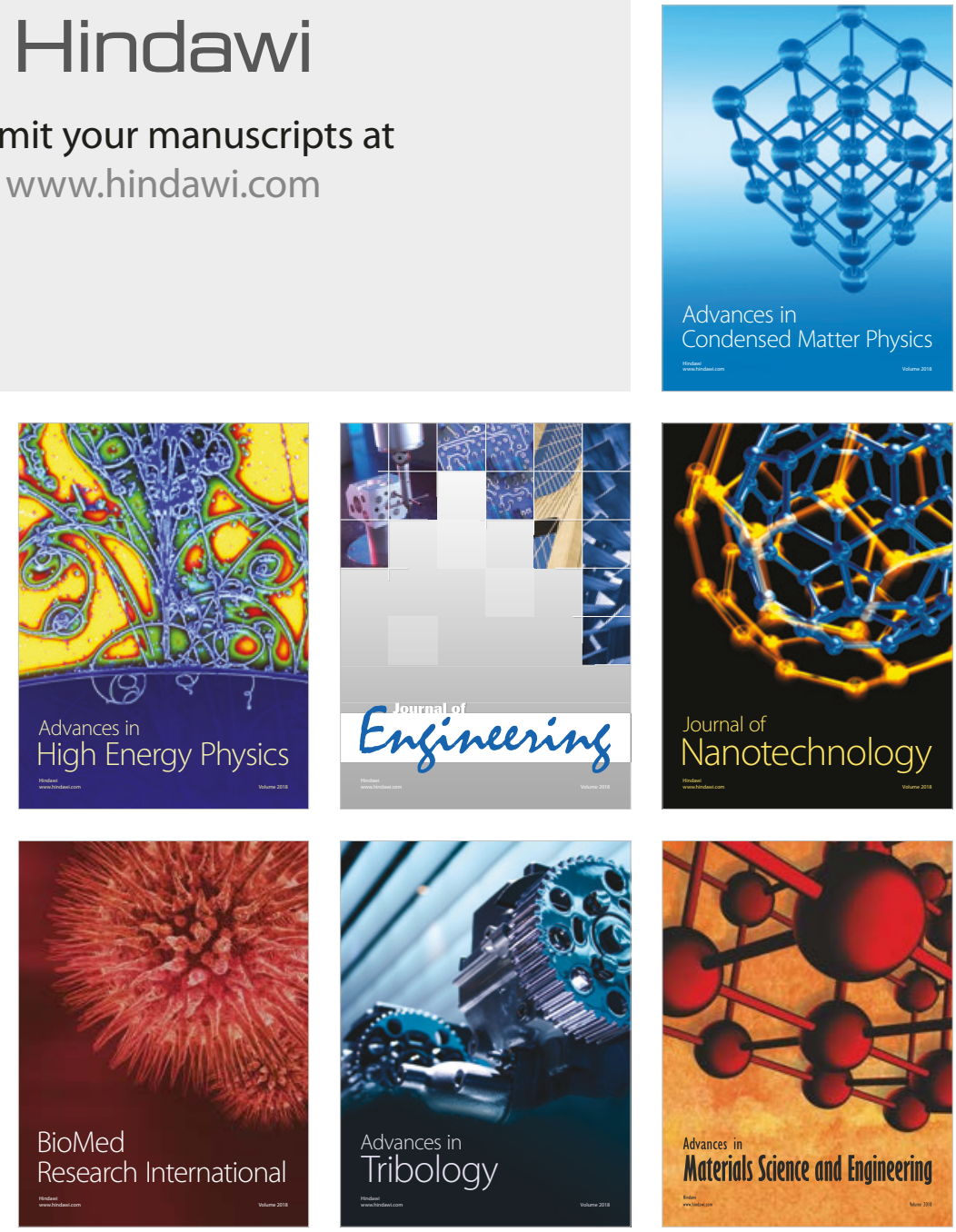